\title{
An unusual case of ruptured noncommunicating rudimentary horn pregnancy at 10 weeks
}

\author{
Kiran Pande*, Angela Yadav
}

Department of Obstetrics and Gynecology, Obstetrics and Gynaecology, MMIMSR, Mullana, Ambala, Haryana, India

Received: 30 November 2019

Revised: 31 December 2019

Accepted: 04 January 2020

\author{
*Correspondence: \\ Dr. Kiran Pande, \\ E-mail: drkiranpande@gmail.com
}

Copyright: $\odot$ the author(s), publisher and licensee Medip Academy. This is an open-access article distributed under the terms of the Creative Commons Attribution Non-Commercial License, which permits unrestricted non-commercial use, distribution, and reproduction in any medium, provided the original work is properly cited.

\begin{abstract}
Unicornuate uterus with noncommunicating rudimentary horn occurs due to incomplete fusion of mullerian ducts. Pregnancy in this horn is a rare phenomenon usually resulting in rupture during second trimester of pregnancy. Prerupture diagnosis of pregnancy in rudimentary horn with ultrasonography is technically difficult, with sensitivity of $30 \%$. We report a case of ruptured non-communicating rudimentary horn at 10 weeks 3 days in a woman. She had a routine USG before pregnancy in which diagnosis was missed. Later she presented to emergency in shock, with massive hemoperitoneum and ruptured horn. So, a high index of suspicion is required to save this catastrophic event and associated maternal morbidity and mortality.
\end{abstract}

Keywords: Laparotomy, Pregnancy, Rudimentary horn, Shock, Trimester, Uterus

\section{INTRODUCTION}

Unicornuate uterus with a rudimentary horn is a rare mullerian anomaly that has a high incidence of obstetric complications that include ectopic pregnancy in the rudimentary horn. ${ }^{1}$ Pregnancy in noncommunicating rudimentary horn is possible by trans-peritoneal migration of sperm or fertilized ovum. It occurs in approximately 1 of every 76,000 pregnancies. The risk of uterine rupture is $50-90 \%$, with most ruptures (approximately 80\%) occurring by the end of the second trimester. ${ }^{1,2}$ We report a case of first trimester rupture of noncommunicating rudimentary horn.

\section{CASE REPORT}

A 23-year-old woman gravida 3, abortion 2 came to emergency with severe lower pain abdomen and vomiting since last two hours. She was 10 weeks pregnant. She denied history of dysmenorrhoea or of pain earlier. The patient had no significant medical or surgical history. On examination patient was in hypovolemic shock with severe pallor, hypotension, and tachycardia. The abdomen was tense and left iliac tenderness was present. Her bowel sounds were normal. On pelvic examination cervix and vagina were healthy, there was no bleeding through os, and size of uterus could not be made out due to intense guarding, left fornix fullness was present. Per rectal examination was within normal limits. Immediately two large bore intravenous cannulas were inserted, one liter of fluid was rushed, patient was catheterized (she passed $50 \mathrm{~mL}$ of clear urine), and urgent investigations and cross match was sent for four units of blood. Her hemoglobin was $5.4 \mathrm{~g} \%$. Outside USG report showed? ruptured ovarian pregnancy one-unit blood was rushed, and after stabilization patient was taken for exploratory laparotomy. Intraoperatively a unicornuate uterus with rupture of left side noncommunicating rudimentary horn which was connected to left ovary and fallopian tube was confirmed (Figure 1) with two liters of hemoperitoneum. 


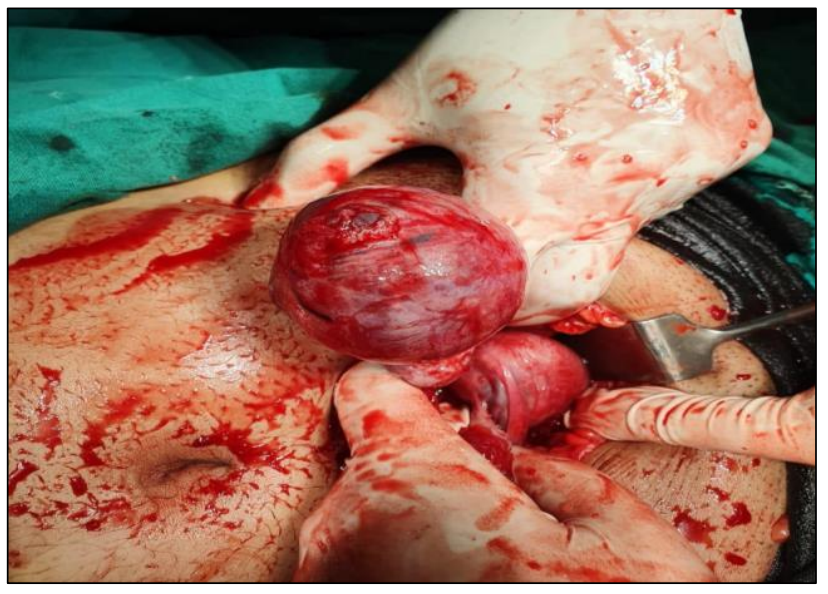

Figure 1: Ruptured non communicating rudimentary horn with gestational sac in situ attached with left ovary. It also shows the unicornuate uterus with right tube and ovary.

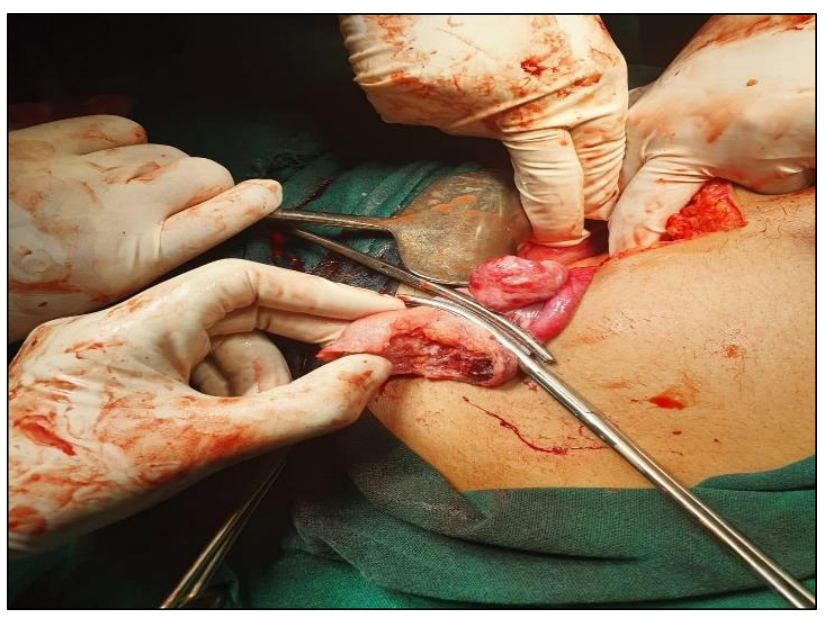

Figure 2: The rudimentary non communicating left horn (after removal of the gestational sac) attached to the left tube and ovary.

Ruptured non communicating rudimentary horn with gestational sac in situ attached with left ovary. It also shows the unicornuate uterus with right tube and ovary

The non-communicating rudimentary horn was not attached to the unicornuate uterus (Figure 2). Both the ovaries and tubes were normal. Left fallopian tube and ovary were attached to the non-communicating horn and there was no communication between the left ovary, tube and the unicornuate uterus. Retroperitoneal ectopic kidney was also confirmed by urosurgeon. Excision of rudimentary horn, ipsilateral left sided salpingectomy was done and sent for histopathology. Left ovary was healthy and preserved. Peritoneal toileting was done. Patient received three units of blood transfusion. She had an uneventful recovery and was discharged on day 7 post-operative.
Intraoperative diagnosis was made as ruptured non communicating horn with hemiuterus class U4a.

Diagnosis was confirmed by biopsy report showing retained products of conception with left rudimentary horn with left fallopian tube.

\section{DISCUSSION}

Mariceau and vassal published the first description of a rudimentary horn pregnancy in 1669, and 600 cases have since been described. ${ }^{3}$ Pregnancies occur in both communicating and noncommunicating horns in proportion to their relative incidence and are equally likely to rupture. ${ }^{2}$ Neonatal mortality is very high as most cases are emergency laparotomies after uterine rupture at premature gestational age. ${ }^{4}$ Maternal mortality is low $(0.5 \%)$ but morbidity is very high in view of massive blood loss and morbidly adherent placentation. ${ }^{2,4}$

The prerupture diagnosis of pregnancy in rudimentary horn has drastically reduced maternal mortality. ${ }^{3}$ But the sensitivity of ultrasound to detect prerupture rudimentary horn pregnancy is very poor $(30 \%)$, probably because of rarity of the diagnosis and nonfamiliarity of the radiologists about this potentially lethal condition. ${ }^{4}$ Early diagnosis before rupture can be managed laparoscopically by immediate excision of the horn, pregnancy, and the ipsilateral fallopian tube.

\section{Tsafrir et al, proposed the following criteria for ultrasonographic diagnosis}

- A pseudo pattern of an asymmetrical bicornuate uterus

- Absent visual continuity tissue surrounding the gestation sac and the uterine cervix

- The presence of myometrial tissue surrounding the gestation sac. In any doubtful case three-dimensional ultrasound or magnetic resonance imaging should be done to avoid the potential complications.

This case highlights the fact that this patient was missed on scan. Hence, this woman was also not warned regarding potential complications. Hence, this case is being reported to familiarize the radiologists regarding this rare but potentially lethal presentation which if diagnosed safely in prerupture state can be managed laparoscopically without the associated sequelae of rupture uterus. Three-dimensional ultrasound imaging and MRI are useful tools in the improvement of diagnostic accuracy, guiding both counseling and surgical planning. ${ }^{3}$

This case further raises the question of whether routine excision of rudimentary horn be undertaken in women with unicornuate uterus as a prophylaxis to prevent such catastrophes. 
A further evaluation of timing of such a surgery is required in a case series, which seems highly unlikely considering the rarity of the condition. In this opinion this decision should be extrapolated from isolated case reports only and routine laparoscopic excision of rudimentary horn with ipsilateral fallopian tube should be offered to these women and those refusing should be adequately counseled regarding the potential complications and if pregnancy occurs in rudimentary horn first trimester laparoscopic excision should be done.

Funding: No funding sources

Conflict of interest: None declared

Ethical approval: Not required

\section{REFERENCES}

1. Rackow BW, Arici A. Reproductive performance of women with müllerian anomalies. Current Opinion Obstet Gynecol. 2007;19(3):229-37.
2. Nahum GG. Rudimentary uterine horn pregnancy: the $20^{\text {th }}$-century worldwide experience of 588 cases. J Repro Med Obstet Gynecol. 2002;47(2):151-63.

3. Fitzmaurice LE, Ehsanipoor RM, Porto $M$. Rudimentary horn pregnancy with herniation into the main uterine cavity. Am J Obstet Gynecol. 2010;202(3):e5-e6.

4. Chopra S, Keepanasseril A, Rohilla M, Bagga R, Kalra J, Jain V. Obstetric morbidity and the diagnostic dilemma in pregnancy in rudimentary horn: retrospective analysis. Arch Gynecol Obstet. 2009;280(6):907-10.

Cite this article as: Pande K, Yadav A. An unusual case of ruptured noncommunicating rudimentary horn pregnancy at 10 weeks. Int J Reprod Contracept Obstet Gynecol 2020;9:839-41. 